\title{
Detection Sensitivity Optimization of Optical Signals Generated by Fiber Optic Bragg Gratings Under Dynamic Excitation
}

John Lekki

James A. Lock

Cleveland State University, j.lock@csuohio.edu

Follow this and additional works at: https://engagedscholarship.csuohio.edu/sciphysics_facpub

Part of the Physics Commons

How does access to this work benefit you? Let us know!

Publisher's Statement

Copyright 2003 Society of Photo-Optical Instrumentation Engineers. One print or electronic copy may be made for personal use only. Systematic reproduction and distribution, duplication of any material in this paper for a fee or for commercial purposes, or modification of the content of the paper are prohibited. Available from publisher at http://dx.doi.org/10.1117/1.1534593.

\section{Original Citation}

Lekki, John and James A. Lock. "Detection Sensitivity Optimization of Optical Signals Generated by Fiber Optic Bragg Gratings Under Dynamic Excitation." Optical Engineering 42 (2003): 425-430.

\section{Repository Citation}

Lekki, John and Lock, James A., "Detection Sensitivity Optimization of Optical Signals Generated by Fiber Optic Bragg Gratings Under Dynamic Excitation" (2003). Physics Faculty Publications. 36.

https://engagedscholarship.csuohio.edu/sciphysics_facpub/36

This Article is brought to you for free and open access by the Physics Department at EngagedScholarship@CSU. It has been accepted for inclusion in Physics Faculty Publications by an authorized administrator of EngagedScholarship@CSU. For more information, please contact library.es@csuohio.edu. 


\section{Detection sensitivity optimization of optical signals generated by fiber optic Bragg gratings under dynamic excitation}

\author{
Grigory Adamovsky \\ National Aeronautics and Space \\ Administration \\ Glenn Research Center at Lewis Field \\ MS 77-1, 21000 Brookpark Road \\ Cleveland, Ohio 44135 \\ E-mail: GAdamovsky@grc.nasa.gov
}

John Lekki, MEMBER SPIE

National Aeronautics and Space Administration

Glenn Research Center at Lewis Field

MS 77-1, 21000 Brookpark Road

Cleveland, Ohio 44135

\author{
James A. Lock \\ Cleveland State University \\ Department of Physics \\ 2121 Euclid Avenue \\ Cleveland, Ohio 44115
}

\begin{abstract}
The dynamic response of a fiber optic Bragg grating to mechanical vibrations is examined both theoretically and experimentally. The theoretical expressions describing the consequences of changes in the grating's reflection spectrum are derived for partially coherent beams in an interferometer. The analysis is given in terms of the dominant wavelength, optical bandwidth, and optical path difference of the interfering signals. Changes in the reflection spectrum caused by a periodic stretching and compression of the grating are experimentally measured using an unbalanced Michelson interferometer, a Michelson interferometer with a nonzero optical path difference. The interferometer's sensitivity to changes in the dominant wavelength of the interfering beams is measured as a function of interferometer unbalance and is compared to theoretical predictions. The theoretical analysis enables the user to determine the optimum performance for an unbalanced interferometer. (c) 2003 Society of Photo-Opticat instrumentation Engineers. [DOI: 10.1117/1.1534593]
\end{abstract}

Subject terms: Bragg gratings; fiber optics; optical sensors; wavelength detection; unbalanced interferometers.

Paper 020135 received Apr. 8, 2002; accepted for publication Jul, 9, 2002.

\section{Introduction}

Fiber optic Bragg gratings (FOBGs) have become a useful tool for sensing applications. ${ }^{1,2}$ They have been successfully used in the validation of the structural integrity of bridges and aircraft components, ${ }^{3-5}$ temperature and pressure measurements in various applications, ${ }^{6-8}$ and detection of leaks. ${ }^{9}$ In addition to their use in static and quasistatic situations, dynamic changes in various system parameters have been measure ${ }^{10,11}$ using an FOBG. In each of these applications, changes in a parameter of the system under observation such as strain or temperature cause a corresponding change in the wavelength of light reflected by the grating. A suitable detector detects the changes in the wavelength and generates corresponding signals that are further analyzed by signal processing electronics.

This paper provides a theoretical foundation for analysis of optical signals reflected by FOBGs under dynamic excitation. In particular, the performance of an unbalanced interferometer used as a spectrometer is analyzed, and the optical path difference producing maximal sensitivity to changes in the wavelength reflected by an FOBG is derived. An unequal optical pathlength interferometer was chosen because of its high sensitivity ${ }^{12}$ as well as its compact size and low weight. These features make unbalanced interferometers especially attractive for in-flight health monitoring of aerospace vehicles and their components.

The paper also provides a detailed derivation of equation used to optimize the design of an unbalanced interferometer for the analysis of spectrally encoded signals. The equations are derived assuming the signals have a Gaussian power spectral density and are consistent with a previously reported analysis (Ref. 13). An experiment was devised to verify the theoretical dependence of the optical path length deference on the detected intensity. A preliminary version of this experiment has been reported elsewhere. ${ }^{14}$ The experimental data are compared to the predicted results and possible causes of the small discrepancy between the two are discussed.

\section{Interference of Two Optical Beams in an Interferometer}

The light intensity produced by the interference of two electromagnetic beams depends on the intensities of the beams and their optical path difference, as well as on the dominant wavelength and bandwidth of each of the beams. Consider a beam entering an interferometer and having a dominant wavelength $\lambda_{0}$ and full width at half maximum optical bandwidth $\Delta \lambda$. After being equally divided by a beamsplitter, the two component beams, each of intensity $I_{0}$, traverse two different paths, of length $l_{1}$ and $l_{2}$, before being recombined at the detector. The optical path difference (unbalance) of the interferometer is $n \Delta l=n\left(l_{1}-l_{2}\right)$. The beams exiting the interferometer interfere at a detector with the intensity

$I=2 I_{0}\left[1+\cos \left(\frac{2 \pi}{\lambda_{0}} n \Delta l\right) \gamma(n \Delta l)\right]$

where $\gamma(n \Delta l)$ is the fringe visibility or fringe contrast reduction factor due to the spread in wavelength of each of 
the beams. We assume the spectral power density $I(v)$ of each beam is Gaussian ${ }^{14}$ and write the expression for $I(v)$ using conventional notation ${ }^{15}$

$I(v)=\frac{2(\ln 2)^{1 / 2}}{\sqrt{\pi} \Delta v} \exp \left\{-\left[2(\ln 2)^{1 / 2} \frac{v-v_{0}}{\Delta v}\right]^{2}\right\}$,

where $v_{0}=c / \lambda_{0}, c$ is the speed of light, and

$\Delta v=c \frac{\Delta \lambda}{\lambda_{0}^{2}}$

The fringe visibility factor is the modulus of the complex degree of coherence, i.e., the Fourier transformation of the of the spectral power density function, ${ }^{15}$

$$
\begin{aligned}
\gamma(\tau)=|\mathfrak{J}[I(v)]| & =\left|\int_{0}^{\infty} I(v) \exp (-j 2 \pi v \tau) \mathrm{d} v\right| \\
& =\exp \left\{-\left[\frac{\pi \Delta v \tau}{2(\ln 2)^{1 / 2}}\right]^{2}\right\} .
\end{aligned}
$$

Using Eq. (3) and Eq. (4), we obtain

$\gamma(\tau)=\exp \left\{-\left[\frac{\pi}{2(\ln 2)^{1 / 2}} \frac{\Delta \lambda}{\lambda_{0}^{2}} c \tau\right]^{2}\right\}$,

and substituting $c \tau=n \Delta l$, the fringe visibility factor can be converted from the time domain to the spatial domain,

$\gamma(n \Delta l)=\exp \left\{-\left[\frac{\pi}{2(\ln 2)^{1 / 2}} \frac{\Delta \lambda}{\lambda_{0}^{2}} n \Delta l\right]^{2}\right\}$.

The intensity of the interference pattern at the detector then becomes

$$
\begin{aligned}
I= & 2 I_{0}\left(1+\cos \left(\frac{2 \pi}{\lambda_{0}} n \Delta l\right)\right. \\
& \left.\times \exp \left\{-\left[\frac{\pi}{2(\ln 2)^{1 / 2}} \frac{\Delta \lambda}{\lambda_{0}^{2}} n \Delta l\right]^{2}\right\}\right) .
\end{aligned}
$$

Figures 1 and 2 show $I / I_{0}$ as a function of the optical path difference for the ranges from 0 to $5 \mathrm{~mm}$ and 2.10 to 2.15 $\mathrm{mm}$, respectively, for $\lambda_{0}=1300 \mathrm{~nm}$ and $\Delta \lambda=0.3 \mathrm{~nm}$. These values are typical of the reflection spectrum of a generic optical communication wavelength FOBG. Note that Fig. 1 contains oscillations that are too fast to resolve. Those oscillations of a cosine nature are clearly seen in Fig. 2.

As the parameters of the interfering beams change, the intensity of Eq. (7) recorded by the detector changes as

$\mathrm{d} I=\frac{\partial I}{\partial(n \Delta l)} \mathrm{d}(n \Delta l)+\frac{\partial I}{\partial \lambda_{0}} \mathrm{~d} \lambda_{0}+\frac{\partial I}{\partial \Delta \lambda} \mathrm{d} \Delta \lambda$.

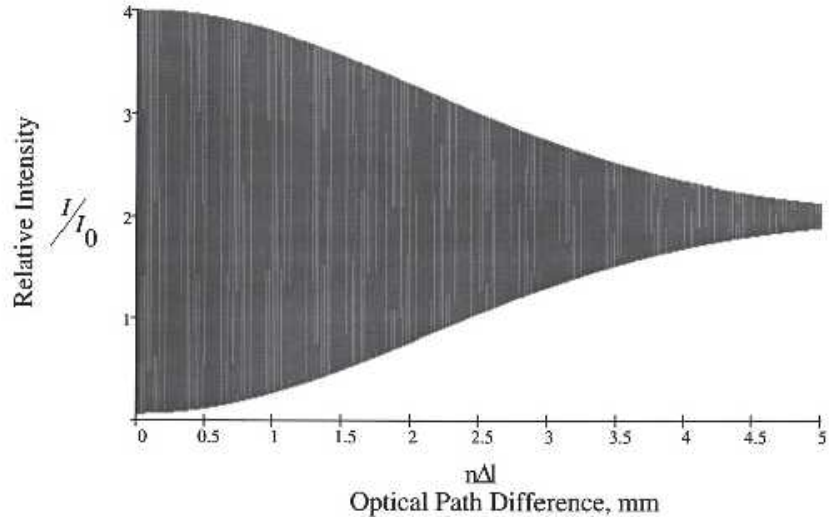

Fig. 1 Intensity of two interfering optical beams of dominant wavelength $\lambda_{D}=1300 \mathrm{~nm}$ and FMHM optical bandwidth $\Delta \lambda=0.3 \mathrm{~nm}$ as a function of optical path difference.

If the optical path difference of the interferometer and the optical bandwidth of the beams remain constant while the dominant wavelength changes, the coefficient

$$
\begin{aligned}
\frac{\partial I}{\partial \lambda_{0}}= & 4 \pi I_{0} \frac{n \Delta l}{\lambda_{0}^{2}}\left\{\sin \left(\frac{2 \pi}{\lambda_{0}} n \Delta l\right)\right. \\
& \left.+\frac{2 \pi}{\lambda_{0}} n \Delta l\left[\frac{1}{2(\ln 2)^{1 / 2}} \frac{\Delta \lambda}{\lambda_{0}}\right]^{2} \cos \left(\frac{2 \pi}{\lambda_{0}} n \Delta l\right)\right\} \\
& \times \exp \left\{-\left[\frac{\pi}{2(\ln 2)^{1 / 2}} \frac{\Delta \lambda}{\lambda_{0}^{2}} n \Delta l\right]^{2}\right\}
\end{aligned}
$$

gives the sensitivity of the intensity recorded by the detector to changes in dominant wavelength of the beams. The first term in curly braces in Eq. (9) is the derivative of the rapid oscillations in Fig. 2 and the second term is the derivative of the much more slowly vary ing overall modulation in Fig. 1. Figures 3 and 4 show the wavelength sensitivity function $S_{\lambda_{0}} \equiv \partial\left(I / I_{0}\right) / \partial \lambda_{0}$ as a function of the optical path difference $n \Delta l$ for the values of $\lambda_{0}$ and $\Delta \lambda$ used in Figs. 1 and 2. Since the second term in curly braces in Eq. (9) is much smaller than the first term, the wavelength sensitivity function can be approximated by

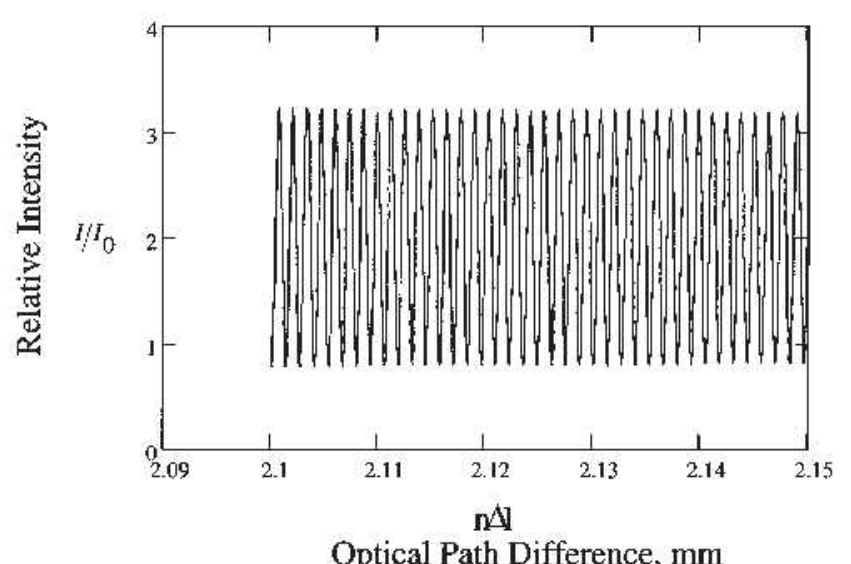

Fig. 2 Fine structure of Fig. 1 for $n \Delta /$ between 2.10 and $2.15 \mathrm{~mm}$. 


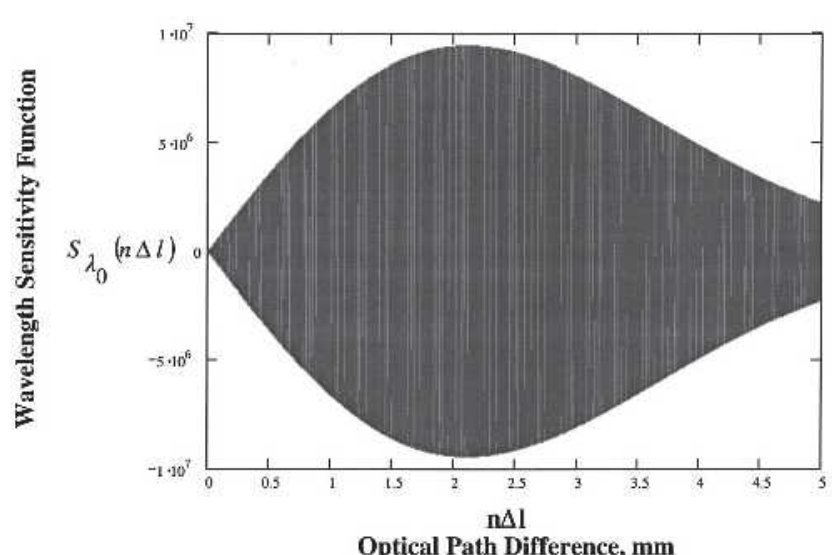

Fig. 3 Interferometer sensitivity to changes in dominant wavelength as a function of optical path difference for $\lambda_{D}=1300 \mathrm{~nm}$ and $\Delta \lambda$ $=0.3 \mathrm{~nm}$

$$
\begin{aligned}
S_{\lambda_{0}}(n \Delta l)= & \frac{4 \pi}{\lambda_{0}} \sin \left(\frac{2 \pi}{\lambda_{0}} n \Delta l\right) \frac{n \Delta l}{\lambda_{0}} \\
& \times \exp \left\{-\left[\frac{\pi}{2(\ln 2)^{1 / 2}} \frac{\Delta \lambda}{\lambda_{0}^{2}} n \Delta l\right]^{2}\right\} .
\end{aligned}
$$

If we had assumed instead that the optical path difference and dominant wavelength remained constant while the optical bandwidth varied, a similar expression for the bandwidth sensitivity function $S_{\Delta \lambda}=\partial\left(I / I_{0}\right) / \partial \Delta \lambda$ could be derived. The sensitivity function $S_{\lambda_{0}}$ in Eq. (10) possesses both rapid oscillatory dependence on $n \Delta l$ and slower linear and exponential dependence. To more easily compare with the experimental results of Sec. 3 , we consider the envelope of the sensitivity function, which we call the envelope sensitivity function,

$E_{\lambda_{0}}(n \Delta l)=4 \pi \frac{n \Delta l}{\lambda_{0}^{2}} \exp \left\{-\left[\frac{\pi}{2(\ln 2)^{1 / 2}} \frac{\Delta \lambda}{\lambda_{0}^{2}} n \Delta l\right]^{2}\right\}$.

The function $E_{\lambda_{0}}$ is graphed as a function of $n \Delta l$ in Fig. 5

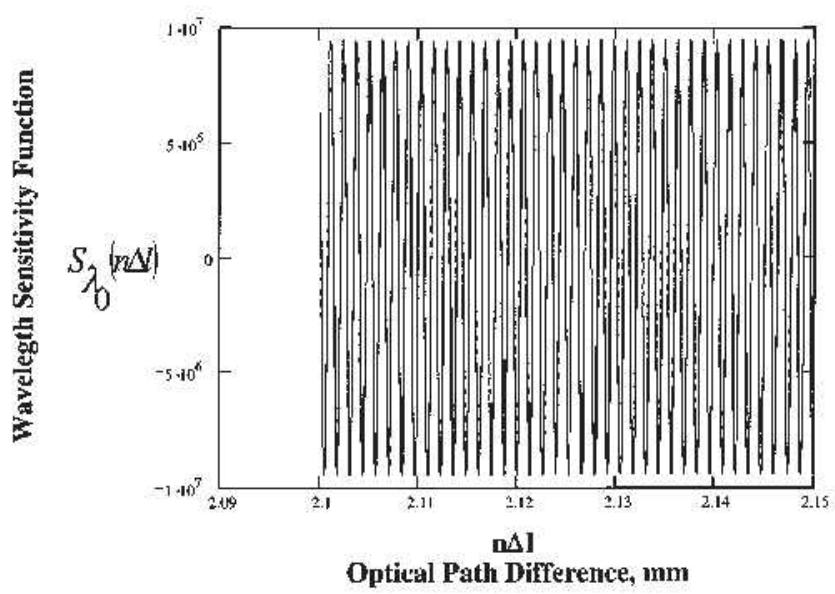

Fig. 4 Fine structure of the wavelength sensitivity function of Fig. 3 for $n \Delta /$ between 210 and $2.15 \mathrm{~mm}$.

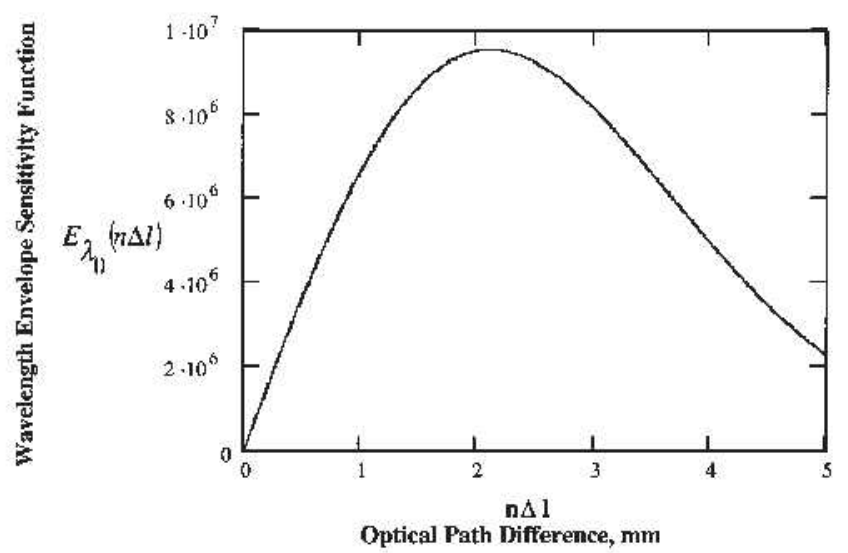

Fig. 5 Wavelength sensitivity envelope $E_{\lambda_{0}}$ as a function of optical path difference for $\lambda_{D}=1300 \mathrm{~nm}$ and $\Delta \lambda=0.3 \mathrm{~nm}$.

for $\lambda_{0}=1300 \mathrm{~nm}$ and $\Delta \lambda=0.3 \mathrm{~nm}$. It is clear from Fig. 5 that to be sensitive to dynamic changes in the dominant wavelength of the signal entering the interferometer, the interferometer must have a nonzero unbalance or optical path difference.

Setting the derivative of $E_{\lambda_{0}}(n \Delta l)$ with respect to the optical path difference to zero, the path difference

$n \Delta l_{\max }=\frac{(2 \ln 2)^{1 / 2}}{\pi} \frac{\lambda_{0}^{2}}{\Delta \lambda}$

produces the maximum sensitivity of a given interferometer to changes in the dominant wavelength of the component beams. For example, for $\lambda_{0}$ and $\Delta \lambda$, as in Figs. 1 to 4 , we obtain $n \Delta l_{\max }=2.111 \mathrm{~mm}$. The dependence of $E_{\lambda_{0}}(n \Delta l)$ and $n \Delta l_{\max }$ on the optical bandwidth is shown in Fig. 6 for $\lambda_{0}=1300 \mathrm{~nm}$. The figure has plots of three curves that describe $E_{\lambda_{0}}(n \Delta l)$ and locations of its maximum that correspond to $n \Delta l_{\max }$ for three different values of $\Delta \lambda$. It could be also observed from Eq. (12) that for a given wavelength $\lambda_{0}$, a decrease in the optical bandwidth $\Delta \lambda$ leads to an

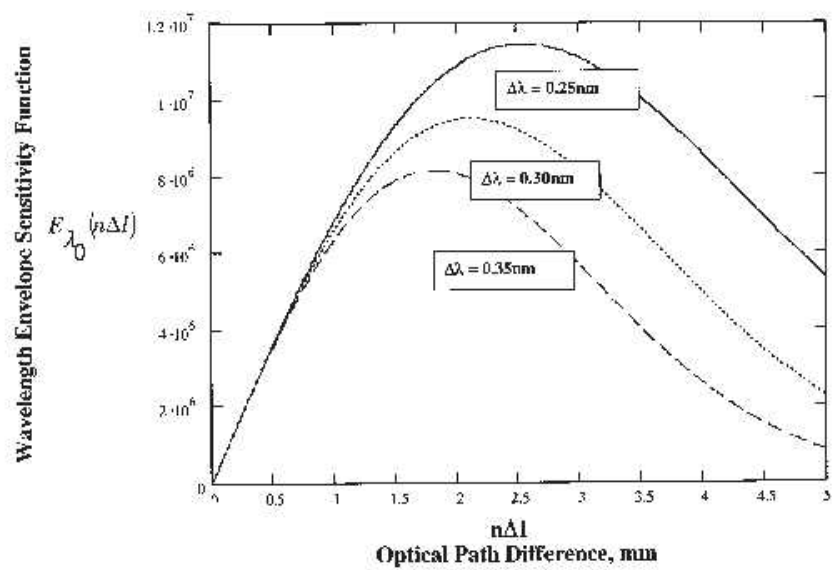

Fig. 6 Wavelength sensitivity envelope $E_{\lambda_{0}}$ as a function of the optical path difference for $\lambda_{D}=1300 \mathrm{~nm}$ and $\Delta \lambda=0.25,0.30$, and 0.35 $\mathrm{nm}$. 
increase in the value of $n \Delta l_{\max }$. Because the bandwidth of optical signal is related to its temporal coherence, highly coherent optical signals would require the interferometer, for $E_{\lambda_{0}}(n \Delta l)$ to reach its maximum, to have a very large unbalance $n \Delta l_{\max }$. Practical considerations, in this case, should be used for the design of an appropriate interferometer.

Since $E_{\lambda_{0}}(n \Delta l)$ in Figs. 5 and 6 is slowly varying near $n \Delta l_{\max }$, it is not necessary to operate the interferometer at exactly this unbalance to achieve good sensitivity. Rather, one can operate the interferometer within a relatively wide range of unbalance about $n \Delta l_{\max }$ and still obtain nearmaximal sensitivity. This range of unbalance depends on the amount of deviation from the maximum sensitivity

$E_{\max }=4\left(\frac{2 \ln 2}{e}\right)^{1 / 2} \frac{1}{\Delta \lambda}$

that can be tolerated. We define the fractional deviation of

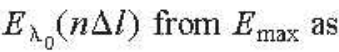

$F=\frac{E_{\max }-E_{\lambda_{0}}(n \Delta l)}{E_{\max }}$.

To determine the range of unbalance for a given $F$ we rewrite Eq. (11), for simplicity, as

$E_{\lambda_{0}}(x)=x \exp \left(-a^{2} x^{2}\right)$,

where $x=4 \pi\left(n \Delta l / \lambda_{0}^{2}\right)$ and $a=1 /\left[8(\ln 2)^{1 / 2}\right] \Delta \lambda$.

The Taylor series expansion of Eq. (15) about $x_{0}$ $=1 /(a \sqrt{2})$ yields

$E(x)=E\left(x_{0}\right)+\left(x-x_{0}\right)\left(\frac{\mathrm{d} E}{\mathrm{~d} x}\right)\left|x_{0}+\frac{\left(x-x_{0}\right)^{2}}{2}\left(\frac{\mathrm{d}^{2} E}{\mathrm{~d} x^{2}}\right)\right|_{x_{0}}$

$$
+\cdots,
$$

where $x_{0}$ is the value of $x$ at which the curve $E_{\lambda_{0}}(x)$ reaches its maximum $E_{\max }=1 /(a \sqrt{2} e)$. The resultant Taylor series in Eq. (16) is approximated by retaining the first two nonzero terms, and after some algebraic simplifications we arrive at

$E(x)=\frac{1}{2 \sqrt{2 e}}\left[1-2\left(x-\frac{1}{a \sqrt{2}}\right)^{2} a^{2}\right]$.

Thus, for $x$ in the vicinity of $x_{0}$, the shape of the curve that describes $E_{\lambda_{0}}(x)$ in Figs. 5 and 6 resembles a parabola. Substituting Eq. (17) into Eq. (14) and rearranging the terms, we obtain

$F=\frac{E\left(x_{0}\right)-E(x)}{E\left(x_{0}\right)}=2 a^{2}\left(x-\frac{1}{a \sqrt{2}}\right)^{2}=\frac{\left(x-x_{0}\right)^{2}}{x_{0}^{2}}$.

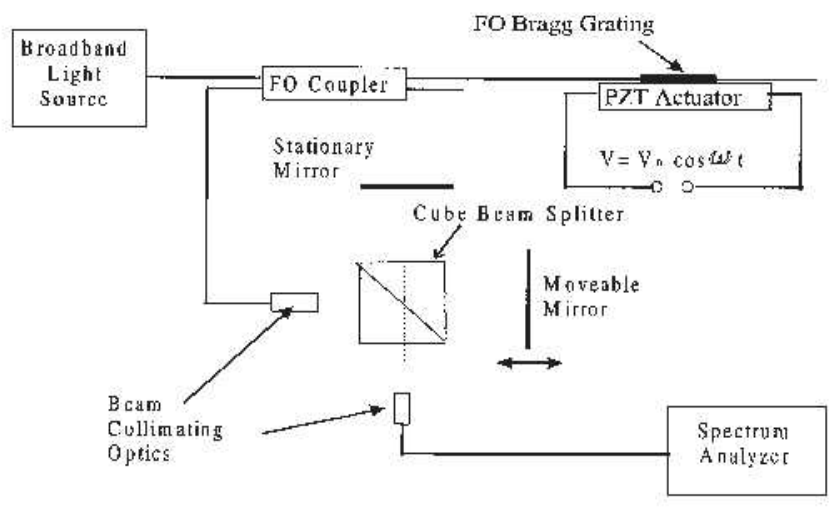

Fig. 7 Experimental apparatus for measuring the wavelength sensitivity envelope function.

Replacing $x$ and $x_{0}$ by the original argument $n \Delta l$ and $n \Delta l_{\max }$, respectively, we yield

$\left|n \Delta l-n \Delta l_{\max }\right| \leqslant \sqrt{F} n \Delta l_{\max }$

for the range of allowable unbalances about $n \Delta l_{\max }$. If, for example, a sensitivity of the interferometer to within $1 \%$ of maximum is desired, an unbalance to within $\pm 10 \%$ of the optimal value is acceptable.

\section{Experiment}

The apparatus of Fig. 7 was used to measure the sensitivity envelope $E_{\lambda_{0}}(n \Delta l)$ of an unbalanced Michelson interferometer. A narrow portion of the $\sim 30$-nm-wide spectrum emitted by a superluminescent diode with the central wavelength of $1300 \mathrm{~nm}$ was reflected by a commercially available telecommunications-grade FOBG and entered the interferometer, consisting of a cube beamsplitter and two mirrors, one of which was mounted on a translation stage. At the beginning of the experiment, the mirrors were positioned at slightly different distances from the respective nearest faces of the beamsplitter. The FOBG was affixed to a lead zirconate titanate piezoelectric element (PZT) and a $1-\mathrm{kHz}$ periodic voltage applied to the PZT generated vibrations that stretched and compressed the grating. The dominant wavelength reflected by the FOBG changed as a function of time in response to the periodic stretching and compression. The resulting time-dependent light intensity exiting the interferometer was recorded by a $p-i-n$ diode and was sent to an electronic spectrum analyzer where the amplitude of the $p-i-n$ diode current at the PZT driving frequency was recorded.

The amplitude of the detected signal at the PZT frequency was measured for a number of different values of interferometer unbalance. Changes in the optical path difference were produced by moving the mirror mounted on the translation stage. Each movement consisted of two steps. First, the mirror was moved approximately $250 \mu \mathrm{m}$ from its previous position. Next, the mirror was moved about this new position several times with an increment of about $0.1 \mu \mathrm{m}$ to determine the maximum amplitude of the $p-i-n$ diode current at the PZT driving frequency. This maximum occurs at the quadrature point of the interferometer, and is proportional to $E_{\lambda_{0}}(n \Delta l)$. The measured wave- 


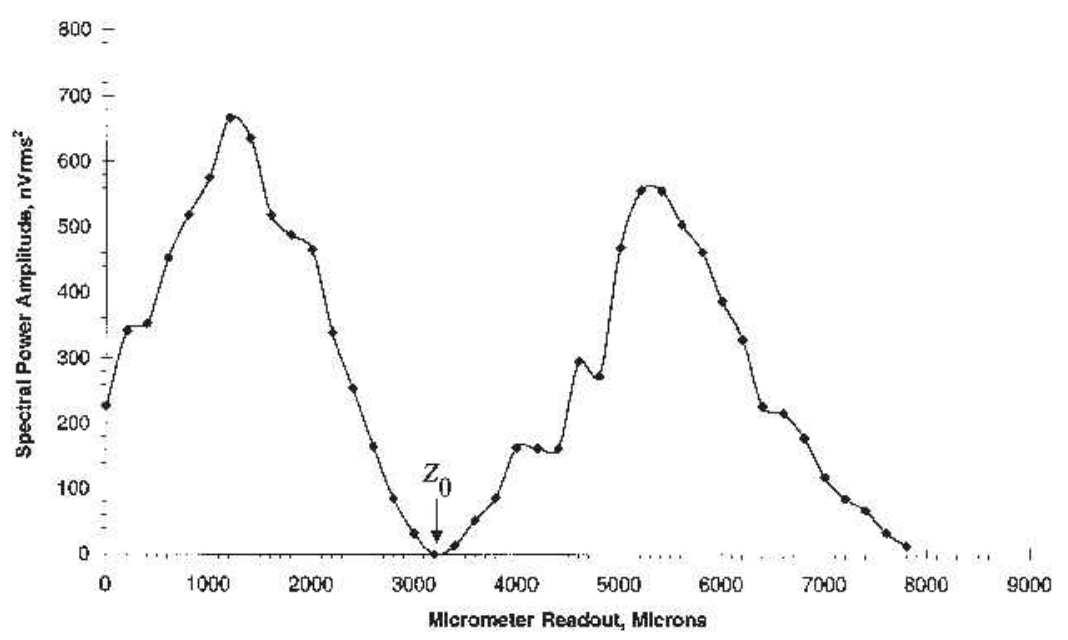

Fig. 8 Measured power at the PZT driving frequency as a function of the micrometer readout of the movable arm of the interferometer.

length sensitivity function is shown in Fig. 8. It is in good qualitative agreement with Fig. 5. Two similarly shaped maximum sensitivity humps in Fig. 8 separated by a point of zero sensitivity are due to the fact that, in the process of changing the optical path difference $n \Delta l$, the movable mirror translated through a point corresponding to a zero optical path difference. This point is denoted in Fig. 8 as $Z_{0}$. An obvious difference between Fig. 5 and the data of Fig. 8 , however, is that although Fig. 5 predicts a linear variation of the sensitivity function near the zero path difference point, the variation in Fig. 8 appears to be roughly quadratic. In addition, the experimental data is more rapidly varying in the vicinity of the relative maximum than is the theoretical prediction. In particular, an optical path length $10 \%$ greater or less than the optimal values in Fig. 8 produces a sensitivity from 7 to $8 \%$ less than maximum, whereas the decrease in the theoretical sensitivity curve of Eq. (11) is only $1 \%$.

To analyze the data of Fig. 8, we assumed the spectrum reflected by the Bragg grating was Gaussian and the optical bandwidth of the reflected spectrum was independent of the dominant wavelength. This last assumption has been found to be accurate for static measurements, ${ }^{16}$ and we presume it is also the case for dynamic excitations of $1 \mathrm{kHz}$. The dominant wavelength of the reflected spectrum of the unstretched grating was determined using an optical spectrum analyzer to be $\lambda_{0}=1310.2 \pm 1 \mathrm{~nm}$, and agreed with the value $\lambda_{0}=1310.17 \pm 0.005 \mathrm{~nm}$ measured at the time of fabrication by the grating manufacturer. The optical bandwidth measured by the manufacturer at that time was $\Delta \lambda$ $=0.192 \pm 0.002 \mathrm{~nm}$. The value of $n \Delta l_{\max }$ corresponding to this optical bandwidth via Eq. (12) is $3.351 \mathrm{~mm}$.

In Fig. 8, the distances between the zero sensitivity point $Z_{0}$ and points of maximum sensitivity to the left and to the right of that point are about $1950 \pm 25 \mu \mathrm{m}$ and $2100 \pm 25$ $\mu \mathrm{m}$, respectively. Because of the double-pass optical configuration of the Michelson interferometer, these distances are equal to half the optical path difference required to achieve maximum sensitivity of the interferometer to changes in dominant wavelength. Thus, the corresponding values of the optimal path difference $n \Delta l_{\max }$ are about 3.9 $\pm 0.05 \mathrm{~mm}$ and $4.2 \pm 0.05 \mathrm{~mm}$ and the corresponding values of optical bandwidth via Eq. (12) are $\Delta \lambda=0.165 \pm 0.002$ $\mathrm{nm}$ and $0.153 \pm 0.002 \mathrm{~nm}$, respectively. The average of these two values of the optical bandwidth is $17 \%$ below that quoted by the manufacturer. A least-squares fit of the results of Fig. 8 to the functional form of Eq. (11) was not attempted.

\section{Discussion}

The good qualitative agreement between the theoretical sensitivity function and the experimental data underscores the correctness of our approach. The relatively small difference between our value of the optical bandwidth obtained experimentally, along with our theoretical prediction of the maximum sensitivity [Eq. (12)], and the value provided by the manufacturer illustrates the basic validity of our theory. There are, however, a number of possible sources of error that could have contributed to the $17 \%$ difference. In particular, the interferometer was not locked at the quadrature position. As a result, slow thermal drifts of the optical path difference or building vibrations could have introduced errors into the measurements. A solution to this would be construction of an unbalanced interferometer on a chip with integrated compensation. Also, in addition to assuming the reflection spectrum of the FOBG was Gaussian and the optical bandwidth was independent of dominant wavelength for dynamic excitations, we also ignored the variation in the $\sim 30$-nm-wide superluminescent diode spectrum over the $\sim 0.2$-nm-wide bandwidth of the FOBG reflection spectrum. This effect, however, is expected to be small.

The slowness of variation of the theoretical sensitivity curve in the vicinity of the relative maximum works to one's advantage when designing an FOBG sensor system. The value of optimum optical difference $n \Delta l_{\max }$ obtained experimentally (Fig. 8) varies roughly from 16 to $25 \%$ of the unbalance $n \Delta l_{\max }=3.351 \mathrm{~mm}$, the value predicted by Eq. (12) using the FOBG manufacturer's optical bandwidth measurement. As a result, one can use Eq. (12) as a reasonable estimate for setting the interferometer unbalance since small deviations of the actual optimal unbalance point from the prediction of Eq. (12) produce correspondingly small 
decreases in the sensitivity of the interferometer to changes in the dominant wavelength of the input signal.

\section{Acknowledgments}

The authors express their gratitude to Mr. Bertram Floyd of AKIMA Inc. for his excellent technical support, and one of the authors (J.A.L.) acknowledges the partial support of the National Aeronautics and Space Administration (NASA) Grant No. NCC3-909.

\section{References}

1. A. D. Kersey, M. A. Davis, H. J. Patrick, M. LeBlanc, K. P. Koo, C. G. Askins, M. A. Putnam, and E. J. Friebele, "Fiber grating sensors," J. Lightwave Technol. 15, 1442-1463 (1997)

2. Y.-J. Rao, "In-fibre Bragg grating sensors," Meas. Sci. Technol. 8, 355-375 (1997)

3. E. Udd, W. L. Schultz, J. Seim, M. Morrell, T. Weaver, J. Bush, and G. Adamovsky, "Fiber optic distributed sensing systems for harsh aerospace environments" in Industrial and Commercial Applications of Smart Strtictures, J. H. Jacobs, Ed., Proc. SPIE 3674, 136-147 (1999).

4. M. Kowalik, I. McKenzie, and S. C. Galea, "Detection of disbands in secondary bonded structures using embedded Bragg grating optical fibre sensors," in Smart Structures and Devices, D. K. Sood, R. A. Lawes, and V. V. Varadan, Eds., Proc. SPIE 4235, 395-406 (2001).

5. S. M. Melle, K. Liu, and R. M. Measures, "Strain sensing using a fibre optic Bragg grating," in Fiber Optic Smart Struc tures and Skins, R. O. Claus and E. Udd, Eds., Proc. SPIE 1588, 255-263 (1991).

6. S. Gupta, T. Mizunami, T. Yamao, and T. Shimomura, "Fiber Bragg grating cryogenic temperature sensors," Appl. Opt. 35, 5202-5205 (1996).
7. Y.-J. Rao, D. J. Webb, D. A. Jackson, L. Zhang, and I. Bennion, "In-fiber Bragg-grating temperature sensor system for medical applications," I. Lightwove Technol. 15, 779-785 (1997).

8. M. G. Xu, H. Geiger, and J. P. Dakin, "Fibre grating pressure sensor with enhanced sensitivity using a glass-bubble housing," Electron Leti. 32, 128-129 (1996)

9. V. V. Spirin, M. G. Shly agin, S. V. Miridonov, E. Mitrani, J. Mendieta, and A. Marquez Lucero, "Fiber optic Bragg grating-based for liquid hy drocarbon leak detection and localization," in Sensory Phenomena and Meastrement Instrtmentation for Smant Strtictures and Materids, R, O. Claus and E. Udd, Eds., Proc. SPIE 3986, 292-299 (2000)

10. S. G. Pierce, W. R. Philp, A. Gachagan, A. McNab, G. Hayward, and B. Culshaw, "Surface-bonded and embedded optical fibers as ultrasonic sensors," Appl. Opt. 35, 5191-5197 (1996).

11. G. Adamovsky, J. Lekki, J. K. Sutter, S. S. Sarkisov, M. J. Curley, and C. E. Martin, "Smart microsystems with photonic element and their applications to aer ospace platform," in Smart Structures and Devices, D. K. Sood, R. A. Lawes, and V. V. Varadan, Eds., Proc. SPIE 4235, $407-418(2001)$

12. A. D. Kersey, T. A. Berkoff, and W. W. Morey, "High-resolution fiber-grating based strain sensor with interferometric wavelength-shift detection," Electron Lett. 28, 236-238 (1992).

13. R. S. Weis, A. D. Kersey, and T. A. Berkoff, "A four-element fiber grating sensor array with phase-sensitive detection," IEEE Photonics Techol. Lett. 6, 1469-1472 (1994).

14. J. D. Lekki, G. Adamovsky, and B. Floyd, "Demodulation system for fiber optic Bragg grating dynamic pressure sensing," in Sensory Phenomena and Me asturement Instrumentation for Smart. Structures and Materials, E. Udd, Ed., Proc. SPIE 4328, 151-159 (2001).

15. J. W. Goodman, Statistic al Optics, Wiley, New York (1985)

16. S. M. Melle, K. Liu, and R. M. Measures, "Practical fiber optic Bragg grating strain gauge system," Appl. Opt. 32, 3601-3609 (1993),

Biographies and photographs of the authors not available. 